\title{
The Design and Implementation of Electromechanical Devices' Operating Parameter Monitoring System in the Highway Tunnel
}

\author{
Hongke $\mathrm{Xu}^{1, \mathrm{a}}$, Xi Zhu ${ }^{2, \mathrm{~b}}$, Guorui $\mathrm{Li}^{3, \mathrm{C}}$ \\ ${ }^{1}$ School of Chang' an University, Xi' an 710000, China; \\ ${ }^{2}$ School of Chang' an University, Xi' an 710000, China; \\ ${ }^{3}$ School of Chang' an University, Xi' an 710000, China; \\ axuhongke@chd.edu.cn, bzhuxichddk@163.com, ${ }^{\mathrm{a}} 710240368 @ q q . c o m$
}

Keywords: Highway Tunnel, Electromechanical Devices, Arduino, ZigBee, Management System

\begin{abstract}
This paper puts forward the highway tunnel electromechanical devices' operating parameter monitoring system is composed of two parts: monitoring network and monitoring management system. Monitoring node puts monitoring data into the database while Monitoring management system chooses MySQL database under B/S architecture by using ExtJS 4.0 and the Struts2 framework. Afterwards, seven function modules has been developed. Finally, the monitoring management system and the monitoring network complete the basic function of the highway tunnel electromechanical devices monitoring system.
\end{abstract}

\section{Introduction}

With the leaping development of China's highway in recent years, the tunnel engineering of highway becomes more and more in the whole highway construction. Meanwhile, the highway tunnel electromechanical system becomes larger and more complex. The way preventing traffic accidents caused by highway tunnel electromechanical devices failure and improving the management level of highway tunnel electromechanical equipment to reduce operating costs become the focus of the highway management departments. Therefore, it is necessary to design electromechanical devices monitoring system of the highway tunnel.

\section{The General Analysis and Design of system}

\subsection{Monitoring objects and Monitoring parameters}

According to the running status of highway tunnel electromechanical system and the relative importance of the subsystems of the highway tunnel electromechanical system, this paper selects key equipments belonging to ventilation system, lighting system, power supply system and traffic monitoring system as the key monitoring equipment of monitoring system. The working parameters of highway tunnel electromechanical devices are mainly composed of environment parameters, electrical parameters and state parameters. Monitoring system chooses the voltage, vibration, temperature and humidity as sampling parameters from electromechanical devices' working parameters, which takes into account the technical feasibility about collecting data and data processing of the monitoring node and experimental conditions.

\subsection{The overall structure of monitoring system}

The function of the monitoring system is to collect the data which is the working parameters of highway tunnel electromechanical devices and transmit the data to monitoring center where there is a monitoring management system manages the data. Therefore, monitoring system consists of two parts, monitoring network based on the monitoring nodes and monitoring management system. The highway tunnel electromechanical devices monitoring system is shown in Figure 1.

Monitoring network is divided into on-site data monitoring network and data communication transmission network. On-site data monitoring network can realize the function of collecting and transmitting the data through the wireless sensor network. Wireless sensor network is a wireless 
network composed by a large number of sensors in the form of self-organization and multiple hops, which can collect and process real-time environmental information and then forward the results to its users. Data communication transmission network is a network where from on-site data monitoring network to remote monitoring center, relying on Ethernet. The gateway note transforms serial data to TCP/IP data which is transmitted through the Ethernet switch and router to the monitoring center. Finally the remote monitoring of highway tunnel electromechanical devices is achieved.

Monitoring management system is constructed with object-oriented program by using the B/S three-tier architecture models including show layer, transaction logical layer and data storage. The development of monitoring management system chooses MySQL database, ExtJS 4.0 and the Struts2 framework with JAVA, JavaScript, etc.

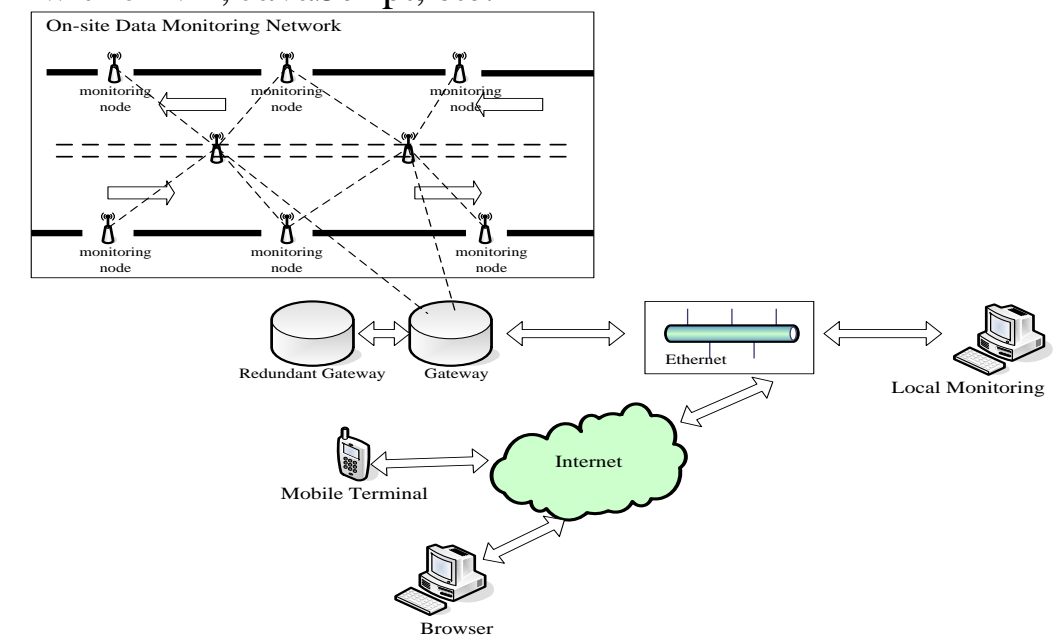

Figure 1. The highway tunnel electromechanical devices monitoring system

\section{The Implementation of system}

\subsection{The implementation of monitoring nodes function module}

Monitoring network based on the monitoring nodes has four function modules: data acquisition, data storage, wireless data transmission and Ethernet communication. The data acquisition includes voltage sampling module, vibration sampling module, temperature and humidity sampling module. Voltage sampling module is composed of DL-PT202D voltage transformer, SX-AC-V quantization module and PCF8591 AD/DA conversion module, whose schematic is shown in Figure 2. YD121-50 piezoelectric accelerometer and PCF8591 AD/DA conversion module are vibration sampling module's two parts, temperature and humidity sensors are AM2313 and three sampling modules' serial interface is the I2C interface. Three sampling modules are read or written by Arduino which has the Wire library and the I2C library.

XBee module which has built-in ZigBee protocol stack can achieve the function of wireless data transmission. XBee has two work modes that are AT and API. Data can be transmitted by XBee's interface and the parameter of XBee is set by X-CTU. Figure 3 is monitoring nodes with XBee module.

The data storage means that data is stored in the EEPROM of Arduino. Arduino is an open source software and hardware platform, which has congenital advantage of solving wireless sensor network and adapting sensor interface. The Arduino UNO, whose core is ATmega328. Schematic of Arduino UNO is chose as shown in Figure 4. 


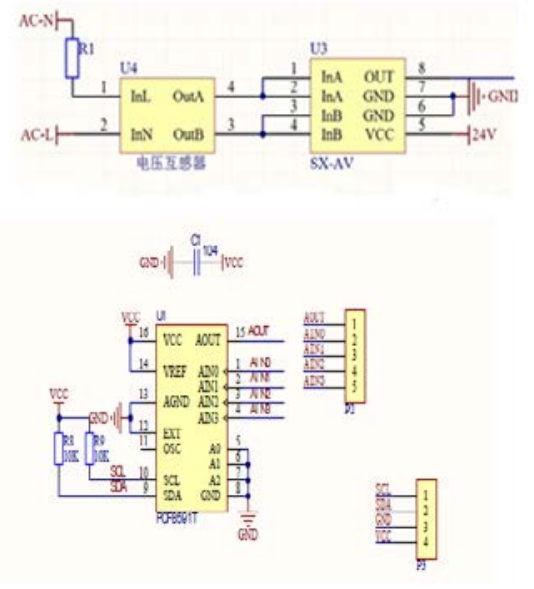

Figure 2. monitoring nodes with XBee modules Figure 3. Schematic of voltage sampling module

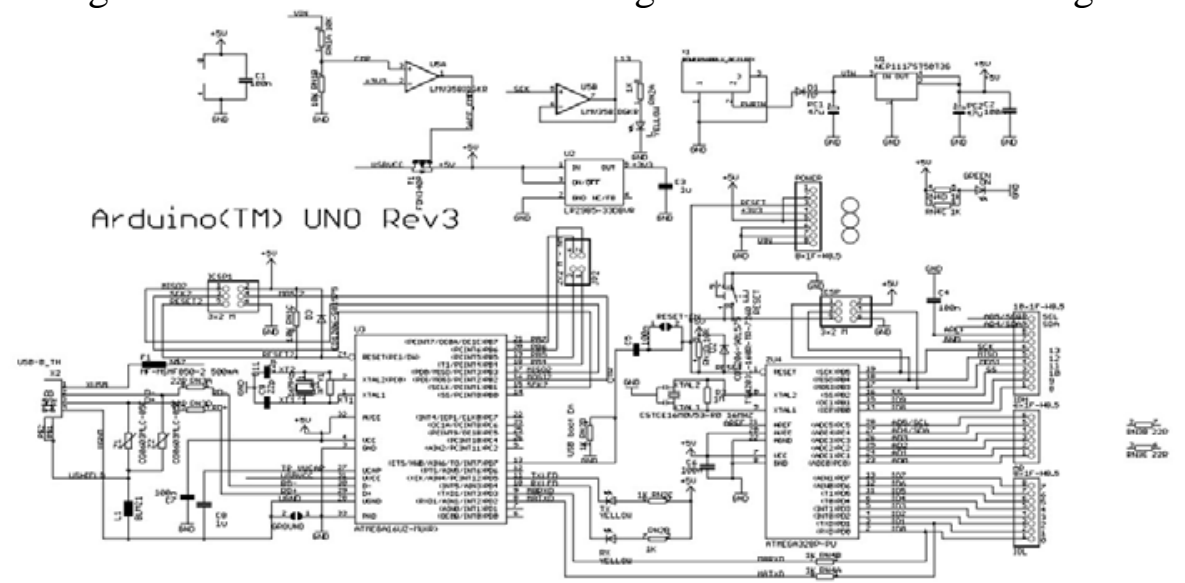

Figure 4. Schematic of Arduino UNO

The function of the Ethernet communication is completed by ENC28J60 with SPI interface. EtherCard can connection network and transmits data which is Arduino's class library. Monitoring note's parameters including MAC address, IP address should be determined as server domain name of a client and data cache. The IP address is acquired automatically and then the GET method which belonging to HTTP protocol is utilized in sending the data to the Web server and putting data into the database through the application.

\subsection{The development of monitoring management system}

Three layers B/S architecture used by monitoring management system is a skeleton of the system, which is popular model structure of a network after the rise of Web, while the Web browser is the most important application of the client. In essence, $\mathrm{B} / \mathrm{S}$ is based on the specific communication protocol (HTTP) of C/S architecture. Three layers of B/S structure can be visualized in figure 5.

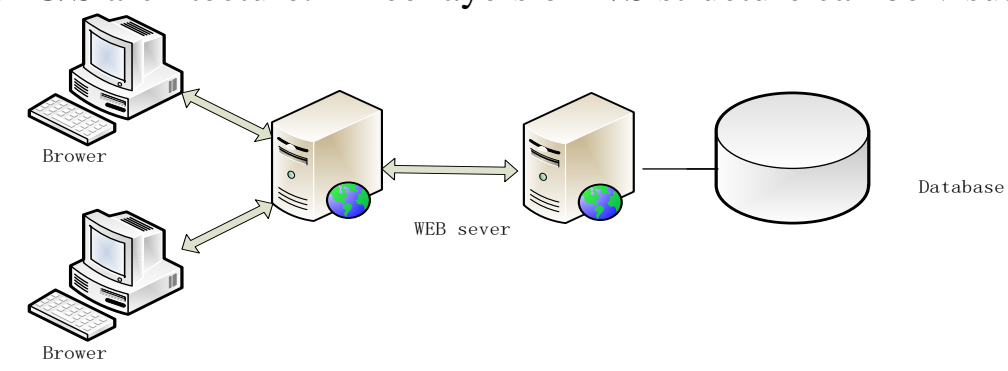

Figure 5. Three layers of B/S structure

Monitoring management system can analysis and monitor sampling data to timely and effectively prevent traffic accidents caused by highway tunnel electromechanical devices failure and makes the highway tunnel electromechanical system run more quickly and effectively. According to the demand analysis, we determine the monitoring management system consists of user login, alarm collection, statistical analysis, real-time monitoring, equipment management, WeChat interaction 
and system configuration. Function block diagram of the whole monitoring system is shown in figure 6.

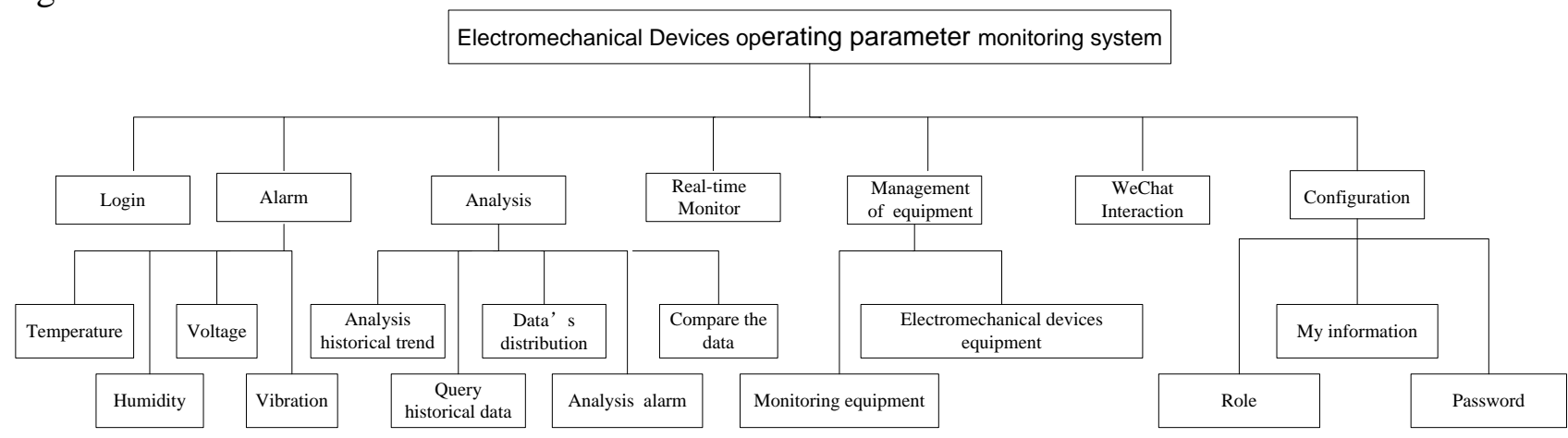

Figure 6. function block diagram of monitoring management system

Seven function modules of Monitoring management system use ExtJS4.0 to achieve the front desk interface development and apply the Struts2 framework for the realization of the background data processing. ExtJS is the front-end Ajax framework which uses JavaScript, CSS and HTML. Struts2 is a MVC (Model View Controller) framework for service on the Web. The Struts2 framework mainly integrates the Struts into Web applications through a filter. Then the Struts2 gets HTTP requests from the Web application-and forwards the HTTP request to the specified Action. Finally, the Action returns related pages to the client according to the result of processing. Limited space, this article only captures three interfaces. Figure 7 is the login interface; and the user must inputs the correct user name and password to enter the system. As shown in Figure 8 for real-time monitor, the peak point is monitor result after humidifying electromechanical devices in the experiment, which reflects the system can real-time monitor the operation condition of electromechanical devices.

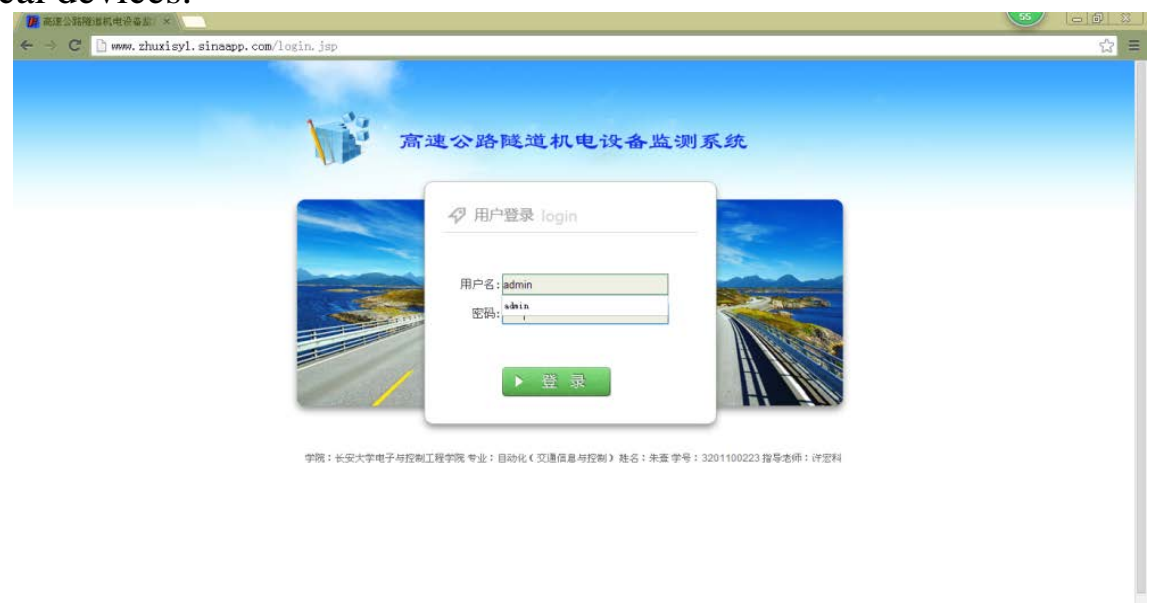

Figure 7. Login

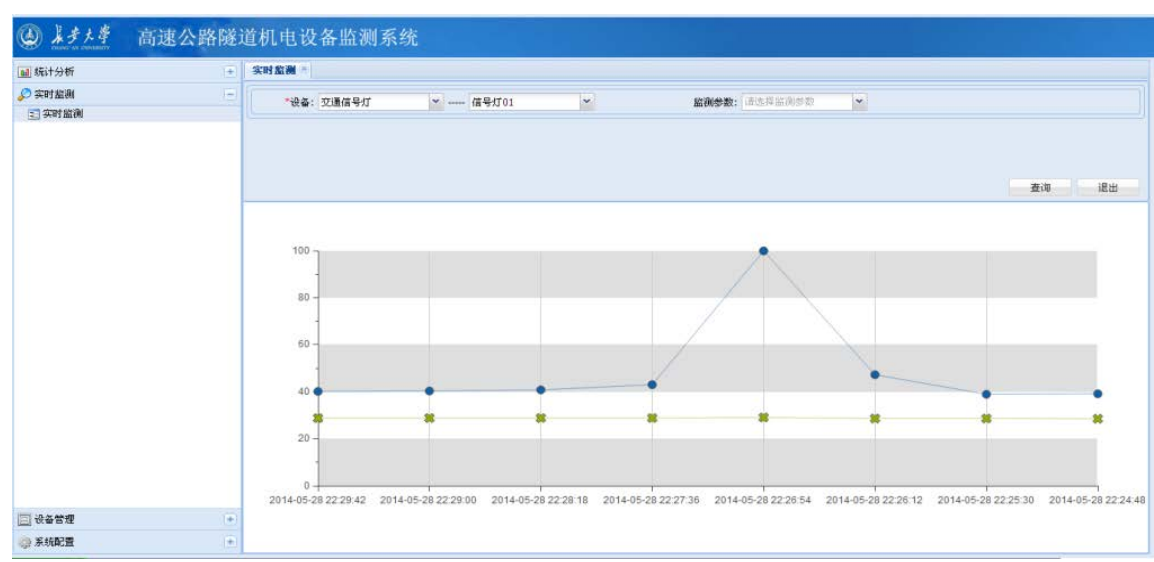

Figure 8. real-time monitor 


\section{Conclusions}

The highway tunnel electromechanical devices' operating parameter monitoring system-composed of monitoring network and monitoring management system is proposed in this paper. Hardware and software of monitoring nodes in monitoring network is designed and meanwhile monitoring management system is developed from four aspects, including functional requirements analysis, architecture design, database design and system implementation. Finally, monitoring system chose the voltage, vibration, temperature and humidity as sampling parameters from electromechanical devices working parameters, which can real-time monitor the operation condition of electromechanical devices and count and analyze the monitoring data.

\section{References}

[1] Zhongjie Zhao, "Mechanical and electrical engineering of highway tunnel”, Beijing:China Communications Press, 2007

[2] Mingxian $\mathrm{Wu}$, "Integrated and intelligent monitoring technology and system of research and development in highway tunnel”, Chang 'an university, 2004

[3] Zheng Shuang, "The transformer running state monitoring system based on wireless sensor network”, Hebei University of Technology, 2012

[4] Cheng Chen, “Arduino development of practical guidelines”, Beijing:Machinery Industry Press,2012

[5] Digi, “XBee/XBee PRO CodeDeveloPment”, DigiInternationalInc,2006

[6] Yinli Jin, "Design and implementation of highway electromechanical equipment maintenance management system”, Highway and Transportation Research,2007(09)

[7] Lei Wu, "Based on B/S rheometer Remote Monitoring System”, Harbin Polytechnic University, 2007

[8] Tomorrow Technology, “Java Web from the entry to the master” Beijing: Tsinghua University Press, 2012

[9] T.Badard, D.Richard, "Using XML for the exchange of updating information between geographical information systems”, Computers, Environment and Urban Systems,2001,25, $25: 17 \sim 31$.

[10]Sams, “Teach Yourself Android Application Development in 24 Hours”, USA: Lauren Darcey\& Shane Conder,2000.1-10. 